EPJ Web of Conferences 19, 02006 (2012)

DOI: $10.1051 /$ epjconf/20121902006

(C) Owned by the authors, published by EDP Sciences, 2012

\title{
On the age of field halo stars
}

\author{
P. Jofré ${ }^{1,2, a}$ \\ ${ }^{1}$ Max-Planck-Institute for Astrophysics \\ ${ }^{2}$ Laboratoire d'Astrophysique de Bordeaux, France
}

\begin{abstract}
A study of stellar ages for a sample from the Sloan Digital Sky Survey (SDSS) is presented. The results are consolidated with a set of globular clusters (GCs) and show that this stellar sample is composed by one dominant population of 10-12 Gyr. This supports the Eggen's scenario, which claims that the inner halo of the Milky Way formed rapidly, probably during the collapse of the proto-Galactic cloud.
\end{abstract}

\section{INTRODUCTION}

There are two main formation scenarios for the Galactic halo. The first one [1] states that the stars in the halo formed slowly and chaotically via merging processes and the second one [2] proposes a rapid formation during the collapse of the proto-Galactic cloud. In the first scenario, the halo is composed by stellar populations of different chemical abundances, kinematics and ages. The product of this chaotic formation has been observed via different evidence [3-5]. In the second formation scenario, the halo is composed by coeval stellar populations, which is supported through studies of GCs [6, 7]. To distinguish between these scenarios and to understand how the Galactic halo might have formed, the ages of field stars play a crucial role. In this report, I present how the ages of halo field stars can be determined.

\section{MAIN SEQUENCE TURN-OFF}

A classical way to determine the age of a star is using isochrones. One has to place the temperature (or color) and the luminosity of the star in the Hertzsprung-Russell Diagram (HRD) and to find the isochrone with the corresponding metal content that crosses this region in the HRD. The determination of the age of an individual halo star is, however, very uncertain because it is almost impossible to place it in a restricted region of the HRD. Firstly, the measurements of atmospheric parameters are uncertain, e.g. an error of $100 \mathrm{~K}$ in the temperature can produce an error of up to $2 \mathrm{Gyr}$ in its age. Secondly, the distance and the mass of halo stars are usually not known, and therefore their intrinsic luminosity is very inaccurate.

The age of halo stars can be studied by considering groups of stars. For coeval stars at a given metallicity, the bluest ones are those at the turn-off point. The age of such a population can then be determined by matching the turn-off temperature to the corresponding isochrone. This study requires independent measurements of metallicities and temperatures of a large sample of stars, from where the turn-off can be determined.

The stellar sample was taken from the SDSS/DR7 data base [8], from which the halo stars were selected using color-color diagrams to focus on the F-G dwarfs and subgiants. The sample contained approximately 100,000 stars with low-resolution spectra, which were used to estimate the metallicity

ae-mail: jofre@obs.u-bordeaux1.fr

This is an Open Access article distributed under the terms of the Creative Commons Attribution-Noncommercial License 3.0, which permits unrestricted use, distribution, and reproduction in any noncommercial medium, provided the original work is properly cited. 


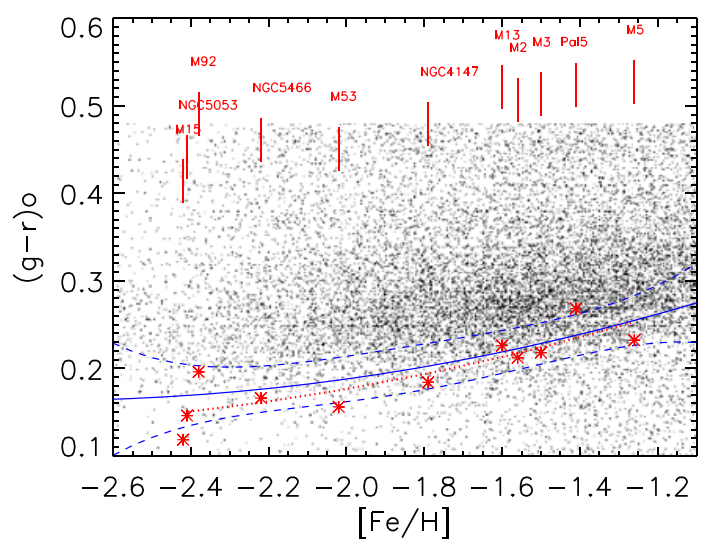

Figure 1. Metallicities and colors of a sample of field halo stars. Red asterisks are the turn-off color and metallicities of globular clusters observed with sloan colors. Blue line represents the turn-off color as a function of metallicity of the field population, with the dashed line its the error.

and temperature. This was done by performing a fitting between the observed spectra and a library of synthetic spectra $[9,10]$.

In a coeval stellar population of a given metallicity, the main-sequence turn-off shows an edge in the temperature distribution function. Figure 1 shows the metallicity and the $(g-r)$ color of the sample. While the number of stars increases smoothly at red colors, at the blue there is an abrupt decrease, which is shown with the solid line. Technically, the abrupt decrease was found by applying the Sobel Kernel [11, and references therein] to the color distribution of the stars at a given metallicity value. The main ingredient of this technique is a first derivative operator that computes the rate of change across an edge, where the largest change corresponds to the edge, i.e. the turn-off. For details on the turn-off detection and its error determination see $[10,11]$. The dashed lines in Fig. 1 represent the errors of the edge detection. Note, there is a significant number of stars below the blue line, which can be blue horizontal branch stars, stars of the same age but metal-poorer, blue stragglers, or younger stars of the same metallicity. It is important to mention that an older population, which would have a redder turnoff color than the dominating population, may well be hidden in this sample. Therefore, the turn-off represented by the blue edge can only estimate the age of the youngest dominant population.

\section{AGE OF THE HALO STARS}

The metallicity and turn-off temperature obtained with the Sobel Kernel technique were used to match the corresponding isochrone. It is important to remark that the absolute age determined using the turnoff temperature can be very sensitive to the effects of gravitational setting $[12,13]$. Here I show only the results obtained from isochrones which include atomic diffusion of the Garching Stellar Evolution Code [14], but an extensive discussion on this subject can be found in [10]. The age as a function of metallicity is illustrated in Fig. 2, where the majority of the stars are 10-12 Gyr old, with no significant age gradient is obtained within the errors.

\subsection{Globular clusters}

GCs can be treated as single stellar populations, where their ages and distances are known with better confidence than those of field stars. Therefore, a sample of GCs with ugriz photometry [15] was used to consolidate the results obtained for the field stars. The Sobel Kernel technique was applied to the $(g-r)$ color distribution of the GC stars in the same way as for the field stars. The blue edge of the 
Assembling the Puzzle of the Milky Way

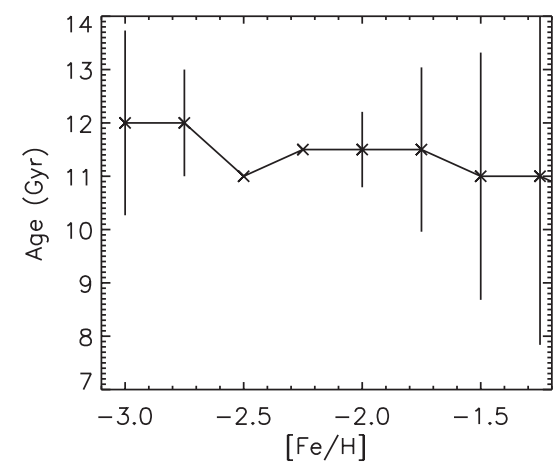

Figure 2. The age as a function of metallicity of the dominant halo-field population.

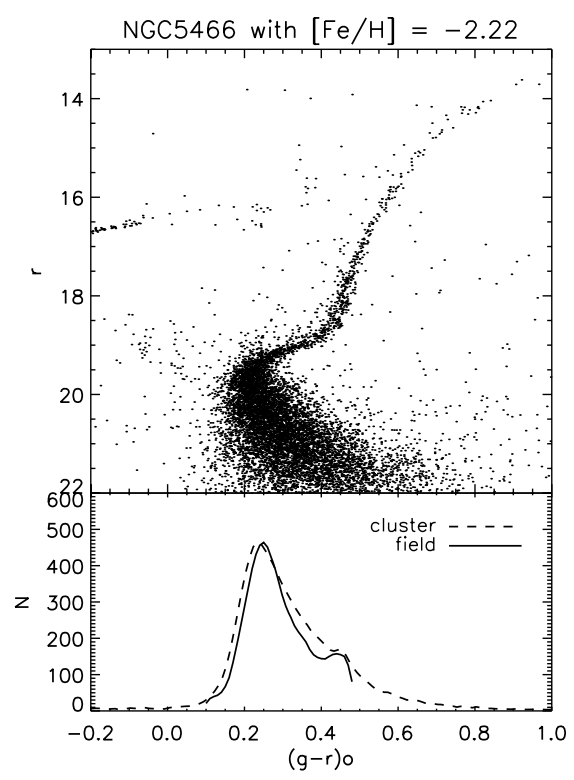

Figure 3. Upper panel: Color-magnitude diagram of an example cluster with ugriz photometry. Lower panel: color distribution of the cluster stars (dashed line) compared with the color distribution of the field stars with the cluster metallicity (solid line).

GCs is plotted in Fig. 1 with asterisks. GC and field stars have the same turn-off color as a function of metallicity, implying that absolute ages should agree too. The study of the absolute age of 55 GCs [6], in which 7 clusters overlap with the present sample, concludes that they are 10-12 Gyr old, showing no gradient of age as a function of metallicity.

Figure 3 shows the color distribution of a GC and the color distribution of the field stars with the corresponding cluster metallicity. The turn-off point is represented by the major peak in the distribution and the second minor peak towards red colors is produced by the stars at the base of the red giant branch. The field stars of the present work must belong to one dominant coeval population because both distributions are bimodal and have similar shapes, and because globular clusters are composed by coeval stars. Moreover, since the turn-off color as a function of metallicity agrees for the field and the cluster stars, they must be coeval to each other. 
EPJ Web of Conferences

\section{DISCUSSION}

The use of independent measurements of spectroscopic temperatures and metallicities implies that the stellar sample is composed mostly by stars belonging to the inner halo. The absence of an age gradient as a function of metallicity found here (see Fig. 2) suggests that there is one dominant population of stars in the inner halo, where the star formation process took place 10-12 Gyr ago.

In addition, there is empirical evidence that cluster and field stars share a similar history and are composed by one dominant population. The colors of sample of clusters observed with SDSS were compared to those of the field stars, where it was shown that both color distributions agreed, especially in the turn-off color as a function of metallicity (see Fig. 3). Moreover, the agreement between the ages for the field stars and for GCs serves as a further argument that the majority of inner halo stars are $10-12$ Gyr old.

An old dominating population for the inner halo gives evidence of a rapid formation of the halo during the collapse of the proto-Galactic gas. This scenario agrees with conclusions from GC studies $[6,7]$, where the metal-poor clusters are coeval. Moreover, the absolute ages obtained for the clusters [6] are also 10-12 Gyr, indicating that GCs and field stars are coeval to each other.

In addition, there is a significant number of stars with bluer colors than the color cut-off of the main sequence turn-off (see Fig. 1), which have also been noticed in similar works [17, 18, and references therein]. One explanation is that they were formed in small external galaxies and have been accreted later on to the Milky Way halo [16]. These galaxies experience a different star formation history to the Galaxy and therefore can have a different age than the dominating population of field stars. Further analysis of their kinematics and chemical abundances is needed to prove this scenario. The blue stars and the existence of a dominant population of halo stars suggest that the two rivaling formation scenarios of the Galactic halo [1,2] actually complete, in a composed manner, the picture of how the Galaxy might have formed. These scenarios combined propose that one part of the halo has collapsed rapidly, while the other part has been populated through collisions and mergers between the satellite galaxies and our Milky Way.

\section{References}

[1] Searle, L. \& Sinn, R., ApJ 225, (1978) 357

[2] Eggen, O. J., Lynden-Bell, D., \& Sandage, A. R., ApJ 136, (1962) 748

[3] Belokurov, V., Zucker, D. B., Evans, N. W., et al., ApJ 642, (2006) 137B

[4] Watkins, L. L., Evans, N. W., Belokurov, V., et al., MNRAS 398, (2009) 1757W

[5] Tolstoy, E., Hill, V., Tosi, M., ARA\&A 47, (2009) $371 \mathrm{~T}$

[6] Salaris, M. \& Weiss, A., A\&A 388, (2002) 492

[7] Sarajedini, A., Chaboyer, B., \& Demarque, P., PASP 109, (1997) 1321

[8] Abazajian et al., K. N., ApJS 182, (2009) 543

[9] Jofré, P., Panter, B., Hansen, C. J., \& Weiss, A., A\&A 517, (2010) A57

[10] Jofré, P., \& Weiss, A., A\&A 533, (2011) 59J

[11] Tabur, V., Kiss, L. L., \& Bedding, T. R., ApJL 703, (2009) L72

[12] Chaboyer, B., Deliyannis, C. P., Demarque, P., Pinsonneault, M. H., \& Sarajedini, A., ApJ 388, (1992a) 372

[13] Salaris, M., \& Weiss, A., ASPC 245, (2001) 367S

[14] Weiss, A. \& Schlattl, H., ApS\&SS 316, (2008) 99

[15] An, D., Johnson, J. A., Clem, J. L., et al., ApJS 179, (2008) 326

[16] Preston, G. W. \& Sneden, C., AJ 120, (2000) 1014

[17] Unavane, M., Wyse, R. F. G., \& Gilmore, G., MNRAS 278, (1996) 727

[18] Schuster, W. J., Moitinho, A., Márquez, A., Parrao, L., \& Covarrubias, E., A\&A 445, (2006) 939 